\title{
Measles high school vaccination program, 2014-2015: online survey of parents in NSW,
} Australia

\section{Sonya Nicholla,c, Holly Seale ${ }^{b}$ and Sue Campbell-Lloyd ${ }^{a}$}

a Immunisation Unit, Health Protection NSW, Sydney, Australia

b School of Public Health and Community Medicine, UNSW Sydney, Australia

c Corresponding author: sonya.nicholl@doh.health.nsw.gov.au

\section{Article history}

\section{Publication date: June 2018}

Citation: Nicholl S, Seale H, CampbellLloyd S. Measles high school vaccination program, 2014-2015: online survey of parents in NSW, Australia. Public Health Res Pract. 2018;28(2):e2821812. https://doi.org/10.17061/phrp2821812

\section{Key points}

- NSW Health sought direct feedback from parents of vaccinated students in a measles high school supplementary immunisation activity (SIA)

- There is limited literature on school-based SIAs that have been implemented as an outbreak response with a short lead time

- The survey results have generated new ideas to further improve delivery of school-based vaccination programs

\section{Abstract}

Background: In 2014, a high school-based measles supplementary immunisation activity (SIA) took place in New South Wales (NSW), Australia, in response to a large number of adolescents being identified as undervaccinated or unvaccinated against measles. The program focused on areas of NSW where previous measles outbreaks had occurred and where large numbers of undervaccinated adolescents lived. More than 11000 students were vaccinated in 2014, and the program continued in 2015, when more than 4000 students in Years 11 and 12 were vaccinated. Parents of students vaccinated during the program were surveyed to determine their level of satisfaction with the program.

Methods: An online link to the anonymous survey with instructions was sent in a text message between August 2015 and May 2016 to parents of students who had consented or been vaccinated during the 2014 and 2015 measles, mumps and rubella (MMR) supplementary immunisation activities (SIAs).

Results: Responses were received from parents in all Local Health Districts (LHDs), and response rates ranged from $<1 \%$ to $21 \%$ across different districts with $59 \%$ of the total number of complete responses from three LHDs. Overall, parents were satisfied with the MMR program, its resources and how it was implemented. Suggestions were received to improve consent processes, increase student involvement and increase school staff accountability. More than half of the parents reported difficulty finding their child's previous vaccination record. Improving vaccination record access and management was highlighted as an area of improvement in the program.

Conclusion: Although response rates were low, the survey has generated important ideas that may help to further improve implementation of school vaccination programs, including allowing electronic consent, increasing student engagement, improving access to previous vaccination records and increasing school staff accountability. 


\section{Introduction}

In 2014, New South Wales (NSW) Health conducted a high school-based measles, mumps and rubella (MMR) supplementary immunisation activity (SIA) in response to a large number of adolescents being identified as undervaccinated (having received only one measlesmumps-rubella [MMR] vaccine dose) or unvaccinated against MMR. ${ }^{1}$ The 2014 MMR SIA was implemented with a very short lead time, and more than 11000 students were vaccinated in 145 high schools in NSW Local Health Districts (LHDs). The SIA focused on students in senior high school years, particularly in South Western Sydney and Western Sydney LHDs that previously had measles outbreaks $^{2}$ (Figure 1). Data from the Australian Childhood Immunisation Register (ACIR) indicated that these LHDs had low vaccination coverage. The program continued in 2015, when more than 4000 students in Years 11 and 12 were vaccinated. Parents received an information kit including the parental consent form, which the students took home from school.

An online survey of parents' perceptions of the NSW school vaccination program had not previously been undertaken. Engaging with parents of students who were vaccinated during the MMR SIA would be likely to generate ideas to further improve the routine schoolbased vaccination program, and improve future SIAs to maximise their impact and increase vaccine uptake.

\section{Methods}

The online survey comprised 10 open- and closedended questions about the MMR SIA. It explored parents' attitudes towards the information provided and the consent process, their record of their child's vaccination, and whether they had any suggestions to improve the program. An online link to the anonymous survey with instructions was sent in a text message to parents of students who had consented or been vaccinated during the 2014 and 2015 MMR SIAs. Between August 2015 and May 2016, the survey link was sent to parents of students approximately 6 months after they were vaccinated during the 2014 and 2015 SIAs. Data were extracted into Excel, cleaned and transferred to SPSS (Armonk, NY: IBM; Version 22). Descriptive statistics of the responses were generated, expressed as absolute and relative frequencies. Free-text fields on suggestions for program improvement were grouped into common categories. The NSW postcode recorded for each child's school was matched to the Australian Bureau of Statistics Socio-Economic Indexes for Areas (SEIFA) decile rank (lowest rank 1 to highest rank 10). ${ }^{3}$ Tests for association between categorical data were carried out using Pearson's chi-squared test $\left(\chi^{2}\right)$ and a $p$ value of $<0.05$ was considered significant.

Figure 1. Location of targeted high schools and MMR vaccine coverage by Local Health District for 11-18-year-olds, 2014

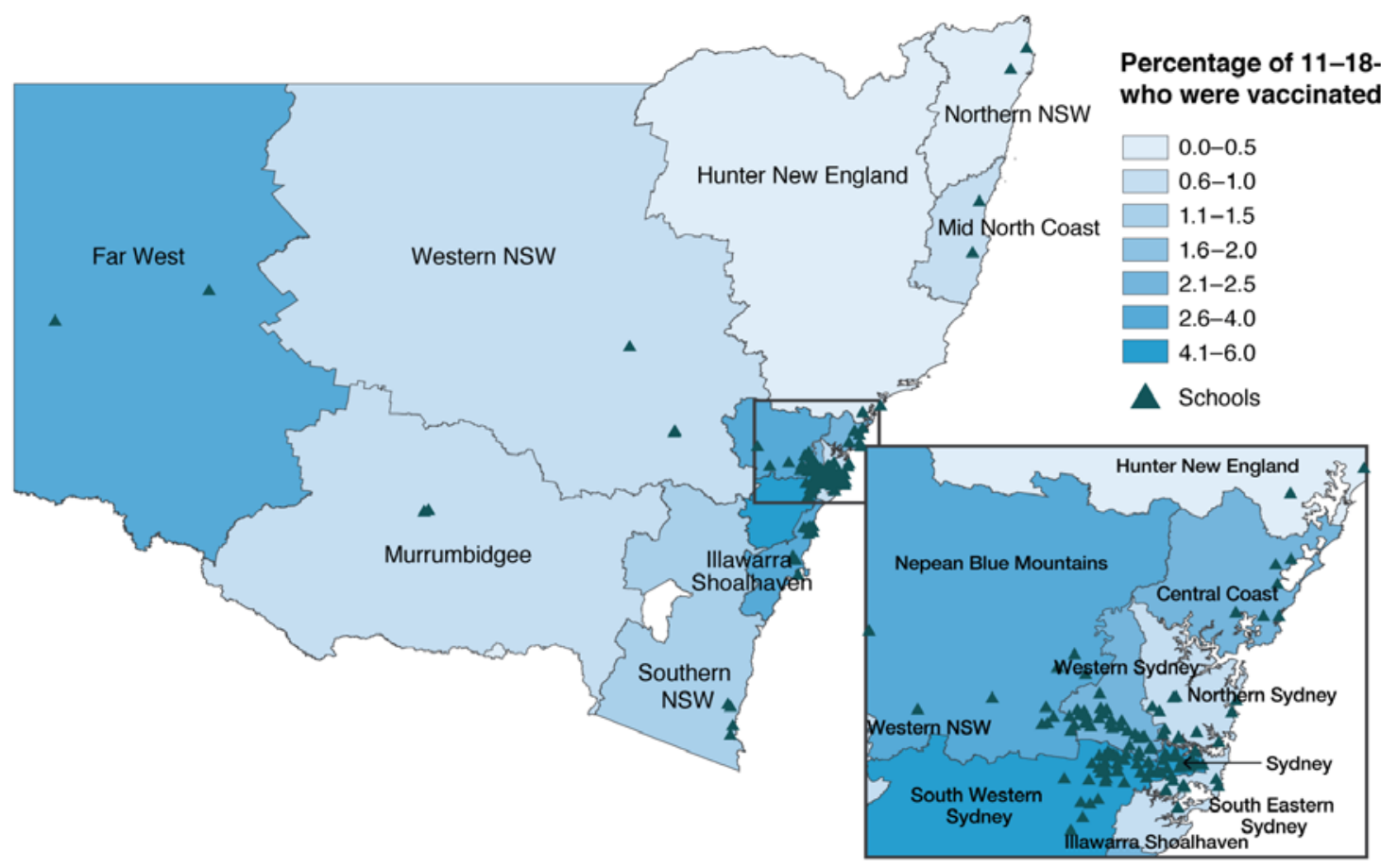

MMR = measles, mumps and rubella; SIA = supplementary immunisation activity

Note: Vaccination coverage data were derived from the Australian Childhood Immunisation Register. 
This work was classified by Health Protection NSW as a service evaluation that did not require ethical approval. The privacy statement in the parent information kit for the SIAs included being contacted afterwards as a service quality improvement initiative. Completion of the survey was accepted as implied consent.

\section{Results}

The survey results are summarised in Tables 1 and 2 . Table 1 provides an overview of response rates. Of the 7878 texts transmitted, 7567 were successfully transmitted, but a small number $(n=311)$ were unable to be transmitted to the mobile phone number listed in the school vaccination database because it was disconnected. A summary of the complete responses to the survey questions $(n=440)$ is provided in Table 2, including questions relating to satisfaction with aspects of the program, student concerns, parental preference for school vaccination program services, and the parents' ability to find their child's previous MMR vaccination record. Figure 2 provides an overview of parent responses by LHD, with response rates ranging from $<1 \%$ to $21 \%$ across different districts; $59 \%$ of the total number of responses came from three LHDs. A quarter of the responses (24\%) came from parents in LHDs where, according to the ACIR, routine childhood immunisation coverage rates appear to be lower than other areas of NSW (Nepean Blue Mountains, Mid North Coast, Northern NSW and Northern Sydney). ${ }^{4}$

Table 1. Summary of survey response rates, August 2015 to May 2016 ( $N=7878)$

\begin{tabular}{lc}
\hline Online survey responses & Response rate \\
\hline Successful text transmissions & $7567(96 \%)$ \\
Unsuccessful text transmissions & $311(4 \%)$ \\
Total responses received & $545(7 \%)$ \\
Complete responses received & $440(6 \%)$ \\
Incomplete responses received & $105(1 \%)$ \\
\hline
\end{tabular}

Table 2. Summary of survey responses to closedended questions, August 2015 to May $2016(n=440)$

\begin{tabular}{|c|c|}
\hline Survey question & $\begin{array}{c}\text { Positive responses } \\
\%\end{array}$ \\
\hline Satisfied with the MMR program & $396(90 \%)$ \\
\hline $\begin{array}{l}\text { Satisfied with parent information kit } \\
\text { advice (contains consent form and } \\
\text { information sheet) }\end{array}$ & $308(70 \%)$ \\
\hline $\begin{array}{l}\text { Satisfied with consent process } \\
\text { (student brings consent form } \\
\text { home for signature) }\end{array}$ & $422(96 \%)$ \\
\hline $\begin{array}{l}\text { Students reported to have brought } \\
\text { their record of vaccination home } \\
\text { to parent }\end{array}$ & $382(87 \%)$ \\
\hline $\begin{array}{l}\text { Student did not express concerns } \\
\text { to parent about receiving MMR } \\
\text { vaccine at school }\end{array}$ & $391(89 \%)$ \\
\hline $\begin{array}{l}\text { School is the preferred place of } \\
\text { vaccination for student (rather } \\
\text { than doctor or community health } \\
\text { centre) }\end{array}$ & $360(82 \%)$ \\
\hline $\begin{array}{l}\text { Difficulty locating student's } \\
\text { previous MMR vaccination history }\end{array}$ & $237(54 \%)$ \\
\hline
\end{tabular}

MMR = measles, mumps and rubella

Note: For the questions relating to participant satisfaction, participants responded to a satisfaction scale of 'very satisfied/satisfied/somewhat satisfied/unsatisfied'. A response of 'very satisfied' or 'satisfied' was considered positive.

Figure 2. Parent responses by Local Health District, August 2015 to May 2016

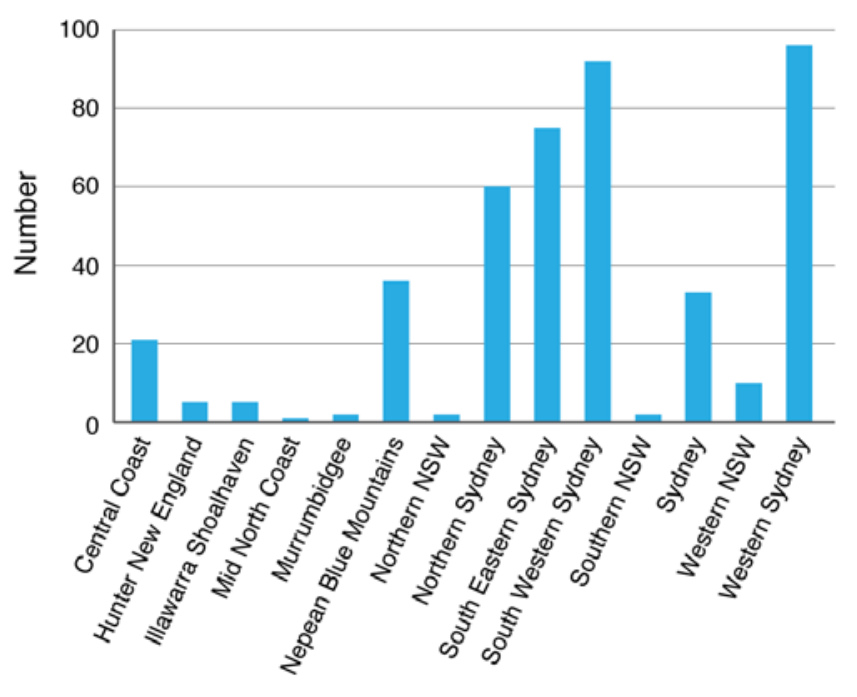

Local Health District 
Overall, $90 \%$ of parents said they were satisfied with the program. Suggestions to improve the school vaccination program are detailed in Box 1. Parents who could easily find their child's records were significantly more likely to have their child bring their vaccination record home $\left(\chi^{2} 8.439, p=0.004\right)$. These parents were also significantly associated with a high satisfaction with the SIA consent processes ( $\left.\chi^{2} 10.744, p=0.001\right)$. A significant relationship was also found between the parents whose first choice for their child to be vaccinated was 'at school' and the school's SEIFA decile rank in NSW $\left(\chi^{2} 11.791, p=0.019\right)$, with parents of children attending a school located in a higher SEIFA decile rank (i.e. higher socio-economic postcode) having the strongest preference for school-based vaccination.

Box 1. Parent suggestions for improving the school vaccination program, August 2015 to May 2016

\section{Consent processes}

- Improve consent processes through the provision of online consent $(n=20)$

- Mandate school vaccinations with an opt-out option $(n=3)$

- Mail a personalised parent information kit directly to parents rather than distribute by students $(n=13)$.

\section{Student engagement}

- Provide an age-appropriate leaflet/advice card to students to ensure the consent form is taken home to parents before the clinic $(n=14)$.

\section{Parental recall and access to previous vaccination records}

- Provide assistance with locating their child's previous vaccination records through advice on where to look $(n=12)$

- Provide access to a state vaccination register $(n=22)$

- Attach a summary of the child's vaccination history to the parent information kit $(n=12)$.

\section{School staff accountability}

- Make school staff more accountable on the day of the vaccination clinic to ensure consented students attend the clinic. Some consented students were not vaccinated because they were not aware the clinic had occurred $(n=10)$.

\section{Discussion}

Although this survey had a low response rate, we believe that the feedback received has generated new ideas for consideration on how to improve school-based vaccination services. Four key areas that were identified by parents for improvement were the consent processes, the need to increase student engagement, the need for better access to previous vaccination records, and the need to increase school staff accountability. Two key ideas for consideration in the future are the importance of using public health staff knowledge when implementing SIAs, and the need to regularly review program implementation through regular parent surveys.

Identifying adolescents for vaccination can be challenging because they form a mobile cohort that does not access medical services frequently. ${ }^{5}$ It is reassuring that school-based vaccination programs are well established in Australia; however, it is important to continually review services, strive for excellence and prevent complacency in school program planning and delivery. Focus groups undertaken with NSW public health unit (PHU) staff (who are responsible for delivering these SIAS) in 2015 identified important considerations for future SIA implementation, such as the importance of adequate lead time and planning time, online consent, student involvement, and facilitating access to schools by the immunisation nurses. ${ }^{6}$ This survey identified a number of improvements for consideration in routine school vaccination program delivery and future SIAs in NSW.

\section{Consent processes}

Ensuring informed consent is an important consideration in the immunisation encounter. ${ }^{7}$ Although parents reported satisfaction with the consent processes and MMR parent information kit, electronic consent has been proposed as an improvement. An electronic approach was also raised during the PHU staff focus groups. ${ }^{6}$ With evolving information technology and national immunisation register developments, this should be pursued further as a potentially efficient and effective tool to obtain informed consent for the NSW school vaccination program and future SIAs.

An opt-out consent approach was also suggested, which has previously been identified as a vaccination consent option. ${ }^{8}$ Although this approach could potentially promote higher vaccine uptake, it may reduce parents' freedom to make an informed choice and it is not consistent with the ethos of healthcare in Australia. ${ }^{9}$ 
It was suggested that providing a personalised parent information kit containing each child's previous vaccination history would assist parents in the consent process. However, using the current information systems, this would be time consuming, logistically difficult and cost prohibitive in routine school vaccination program delivery. ${ }^{10}$ It could, however, be considered for future locally targeted SIAs with smaller numbers.

\section{Student engagement}

In this survey, it was suggested that students should be involved in the consent process so that they understand the importance of the vaccine they are to receive. Vaccine uptake in the program relies on students bringing the parent information kit home to their parent to sign the consent form and also returning the signed consent form to the school. It was suggested that students should be involved in the consent process with their parents and be provided with information about the importance of the vaccine, and this may increase the likelihood that students will deliver the consent form to their parents. Introducing adolescent education and participation in the vaccination decision is important because this may positively influence vaccination decisions for their own children in future ${ }^{11}$ and promote acceptance of future adult vaccinations. Because many adolescents have no experience or memory of vaccine-preventable diseases ${ }^{11,12}$, schools are appropriate settings for educational programs on the importance of vaccination. ${ }^{13}$ Involving adolescents in the consent process may increase vaccine uptake. ${ }^{8}$ Increasing student involvement in the consent process was suggested by parents in this survey and during the NSW PHU focus groups. ${ }^{6}$

\section{Parental recall and access to previous vaccination records}

More than half of the parents (54\%) reported difficulties finding their child's previous MMR vaccination records, which supports unpublished findings from implementing the 2014 and 2015 MMR SIAs. Although more than 11000 were vaccinated in the 2014 SIA, a further 8000 students had parental consent and were not vaccinated; in the 2015 SIA, 20000 students had parental consent and only 4200 students required a vaccine. ${ }^{14}$ The discordance between the consent and vaccination rates occurred because parents who were unsure of their child's vaccination history or were unable to find their vaccination record consented for their child to be vaccinated. A number of PHUs conducted preprogram checks on the ACIR for previous MMR vaccine doses in consenting students where parents indicated that no doses or only one dose had previously been administered. Where a consenting student had a record of two previous MMR vaccine doses on the ACIR, they were not vaccinated during the SIAs. Complete previous vaccination was found for $91 \%$ of the preprogram checks, indicating that the majority of students who consented were already fully immunised against MMR. ${ }^{14}$ The expansion of the national childhood immunisation register to the Australian Immunisation Register (AIR) in October 2016 to capture vaccination records at any age should help parents in the future, because they will have access to their child's complete vaccination history.

High-quality measles coverage data are essential to guide decision making to progress towards measles elimination ${ }^{15}$, and to continue monitoring at-risk groups to identify immunity gaps and community engagement strategies. Immunisation program planners should use the new AIR to identify immunity gaps in population groups and target future SIAs. However, the ability of the AIR to identify immunity gaps in older children and adults is unclear at this stage.

\section{School staff accountability}

Despite parental consent, it was reported that not all students attended the vaccination clinics because some teachers had not informed the students that the clinic had started or the students did not hear the clinic announcement. It was suggested that teachers should be more accountable. The suggestion of increasing school staff accountability is useful, and PHUs should request that their school coordinator cross-reference student attendance at the clinic with each class list, taking into account absences on the day. Across all LHDs, it has previously been informally reported that school staff involvement varies across the district. Some schools have adopted a student checking mechanism and others have not. PHU focus groups identified that a good rapport between the PHU and the school coordinator facilitated program implementation. ${ }^{6}$ It is recommended that PHUs develop a greater rapport with school staff to increase support.

\section{Vaccination program planning}

Socio-economic status (SES) has been found to be an important predictor of MMR vaccine uptake. In some overseas studies, a low school and parent SES was associated with low vaccination coverage. ${ }^{16}$ Demographic factors such as parental education level and income have also been found to affect vaccine uptake. ${ }^{17}$ In this survey, attendance at a school with a high SEIFA decile rank (high SES) was significantly associated with parents' first choice for their child to be vaccinated in the school vaccination program. This highlights the need to ensure that consent materials are pitched appropriately, available in many languages, and received and understood by parents and students in all NSW locations, particularly in areas where vaccine uptake tends to be low. In the current NSW Adolescent Vaccination Program, materials are available in 23 community languages; however, it is important to communicate the availability of these resources to school staff and parents. Actions to 
improve MMR vaccine uptake should focus on families of lower SES, larger families and children of single-parent families. ${ }^{18}$ It is acknowledged that catch-up immunisation campaigns may require a different planning approach to routine campaigns ${ }^{19}$, and, due to the heterogeneous nature of immunisation barriers, tailored interventions are needed ${ }^{20}$ to increase vaccine uptake. The MMR SIA relied on PHU staff knowledge to target specific schools that were identified as having many adolescents who were undervaccinated against measles. Locally targeted SIAs should continue to include PHU staff knowledge on the targeted cohort during planning to achieve high coverage, equitable access and vaccination of hard-toreach individuals.

\section{Program innovation}

Regularly reviewing services is a useful tool to improve program delivery. The recommended vaccination schedule for adolescents may change as a result of advances in vaccine development and emerging vaccine-preventable disease outbreaks, and additional vaccines may be recommended for adolescents for delivery through the school vaccination program in the future. Barriers and facilitators to implementing school vaccination programs in Australia have previously been identified $^{16}$; however, there is a lack of research on the determinants of nonconsent and subsequent influences on parents' decisions. Invigorating school vaccination services through ongoing parent engagement and empowerment in all areas in NSW could facilitate optimal vaccine uptake. It is equally important to continually improve the school vaccination service and identify any barriers to parental consent. It is recommended that regular surveys of high school students' parents are conducted, and that future studies focus on barriers to parental consent in the school vaccination program.

\section{Limitations}

The overall response rate of $7 \%$ means the survey results are not generalisable. The responses received were from parents who consented for their child to be vaccinated, and who would therefore be generally supportive of school vaccination services. Parents received the survey link approximately 6 months after their child was vaccinated, and this may have affected the results because it relied on parents remembering detailed information about the SIA. Only parents who had a mobile phone could receive the survey link, and it is possible that parents who did not provide a mobile phone number or did not have a mobile phone may have different views from those that were contactable. Furthermore, some parents who received the survey link may not have had access to the internet. However, given that $86 \%$ of NSW households had access to the internet in 2014-15, this is not likely to have had a major impact on responses. ${ }^{21}$ Parents who did not have internet access may also be systematically different from the parents who were able to participate.

\section{Conclusion}

Despite the limitations of the survey and low response rate, the survey results provide some reassurance that the NSW school vaccination program satisfies participating parents and students. It has generated ideas for consideration to improve future SIAs and routine school vaccination programs, such as electronic consent, increasing student engagement, improving access to previous vaccination records and increasing school staff accountability. It is recommended that a student advice card should be developed to improve student engagement in the school vaccination program. Future SIAs should be tailored to the targeted cohort using local health professional intelligence and strategies to achieve high vaccination coverage to reduce the risk of further outbreaks of vaccine-preventable diseases.

\section{Peer review and provenance}

Externally peer reviewed, not commissioned

\section{Competing interests}

HS has received payment from bioCSL, GSK and Sanofi Pasteur for lectures, and payment for consultancy services from Sanofi Pasteur. Her institution has received funding for investigator-driven research from bioCSL, GSK and Sanofi Pasteur. These activities were outside the work described in this manuscript. SN and SC-L declare no competing interests.

\section{Author contributions}

SN developed the survey tool and administered the survey. SN analysed the results, which HS cross-checked. SN prepared the manuscript and HS and SC-L provided feedback. SN prepared the manuscript revisions in response to the peer reviewers' feedback.

\section{References}

1. NSW Health. Sydney: State of New South Wales NSW Ministry of Health; 2015. New high school catch up program for measles, mumps and rubella; 2014 Jun 26 [cited 2017 Dec 15]; [about 2 screens]. Available from: www.health.nsw.gov.au/news/Pages/20140625_00.aspx

2. Najjar Z, Hope K, Clark P, Nguyen O, Rosewell A, Conaty S. Sustained outbreak of measles in New South Wales, 2012: risks for measles elimination in Australia. Western Pac Surveill Response J. 2014;5(1):14-20. 
3. Pink B, Australian Bureau of Statistics. Socioeconomic indexes for areas (SEIFA): 2011. Canberra: Commonwealth of Australia; 2013 [cited 2017 Dec 15]. Available from: www.ausstats.abs.gov.au/ausstats/ subscriber.nst/0/22CEDA8038AF7A0DCA257B3B00 116E34/\$File/2033.0.55.001\%20seifa\%202011\%20 technical\%20paper.pdf

4. My Healthy Communities. Canberra: Australian Institute of Health and Welfare; 2018. Immunisation rates for children by Primary Health Network area, 2012-13. Sydney: NHPA; 2014 [cited 2017 Dec 20]. Available from: www. myhealthycommunities.gov.au/interactive/ic01

5. Ward KF, Menzies RI, Quinn HE, Campbell-Lloyd S. School-based vaccination in NSW. N S W Public Health Bull. 2010;21(9-10):237-42.

6. Nicholl S, Seale H, Sheppeard V, Campbell-Lloyd S. Measles prevention in adolescents: lessons learnt from implementing a high school catch-up vaccination programme in New South Wales, Australia 2014-2015. Western Pac Surveill Response J. 2016;73(3).

7. Lopalco P, Sprenger M. Do European doctors support measles, mumps, rubella vaccination programmes enough? Euro Surveill. 2011;16(39).

8. Robbins SC, Bernard D, McCaffery K, Skinner SR. 'It's a logistical nightmare!' Recommendations for optimising human papillomavirus school-based vaccination experience. Sex Health. 2010;7(3):271-8.

9. Mossialos E, Wenzl M, Osborn R, Sarnak D, editors. 2015 international profiles of health care systems, 2015. New York City: The Commonwealth Fund; 2016 [cited 2017 Dec 15]. Available from: www.commonwealthfund. org/ /media/files/publications/fund-report/2016/jan/1857_ mossialos_intl_profiles_2015_v7.pdf

10. Limper HM, Burns JL, Lloyd LM, Atilano J, Alexander KA, Caskey RN. Challenges to school-located vaccination: lessons learned. Pediatrics. 2014;134(4):803-8.

11. Hilton S, Patterson C, Smith E, Bedford H, Hunt K. Teenagers' understandings of and attitudes towards vaccines and vaccine-preventable diseases: a qualitative study. Vaccine. 2013;31(22):2543-50.
12. Esposito S, Principi N, Cornaglia G, ESCMID Vaccine Study Group (EVASG). Barriers to the vaccination of children and adolescents and possible solutions. Clin Microbiol Infect. 2014;20:25-31.

13. Lindley MC, Boyer-Chu L, Fishbein DB, Kolasa M, Middleman AB, Wilson $\mathrm{T}$, et al. The role of schools in strengthening delivery of new adolescent vaccinations. Pediatrics. 2008;121 Suppl 1:S46-54.

14. Wickens M. Concordance of parental recall with Australian Childhood Immunisation Register measles, mumps and rubella data. Sydney: NSW Health; 2015 [unpublished data].

15. Durrheim DN, Crowcroft NS, Strebel PM. Measles - the epidemiology of elimination. Vaccine. 2014;32(51):68803.

16. Cooper Robbins SC, Ward K, Skinner SR. School-based vaccination: a systematic review of process evaluations. Vaccine. 2011;29(52):9588-99.

17. Brown KF, Kroll JS, Hudson MJ, Ramsay M, Green J, Long SJ, et al. Factors underlying parental decisions about combination childhood vaccinations including MMR: a systematic review. Vaccine. 2010;28(26):423548.

18. Vandermeulen $\mathrm{C}$, Roelants $\mathrm{M}$, Theeten $\mathrm{H}$, Van Damme $\mathrm{P}$, Hoppenbrouwers K. Vaccination coverage and sociodemographic determinants of measles-mumpsrubella vaccination in three different age groups. Eur $\mathrm{J}$ Pediatr. 2008;167(10):1161-8.

19. Brown K, Fraser G, Ramsay M, Shanley R, Cowley N, van Wijgerden J, et al. Attitudinal and demographic predictors of measles-mumps-rubella vaccine (MMR) uptake during the UK Catch-Up Campaign 2008-09: cross-sectional survey. PloS One. 2011;6(5):e19381.

20. Pearce A, Marshall H, Bedford H, Lynch J. Barriers to childhood immunisation: findings from the Longitudinal Study of Australian Children. Vaccine. 2015;33(29):337783.

21. Australian Bureau of Statistics. Household use of information technology, Australia 2014-15. Canberra: Commonwealth of Australia; 2016 [cited 2017 Dec 15]. Available from: www.abs.gov.au/ausstats/abs@.nsf/ $\mathrm{mf} / 8146.0$

\section{Copyright: (c)}

(C) 2018 Nicholl et al. This article is licensed under the Creative Commons Attribution-NonCommercial-ShareAlike 4.0 International Licence, which allows others to redistribute, adapt and share this work non-commercially provided they attribute the work and any adapted version of it is distributed under the same Creative Commons licence terms. See: www.creativecommons.org/licenses/by-nc-sa/4.0/ 